\title{
Rehabilitation Planning of Water Distribution Network through a Reliability-Based Risk Assessment
}

\author{
Marianna D'Ercole ${ }^{1, *}$, Maurizio Righetti ${ }^{1}$, Gema Sakti Raspati ${ }^{2}$, Paolo Bertola ${ }^{3}$ and \\ Rita Maria Ugarelli ${ }^{2,4}$ \\ 1 Faculty of Science and Technologies, Free University of Bozen, 39100 Bolzano, Italy; \\ Marianna.Dercole@natec.unibz.it; Maurizio.Righetti@unibz.it \\ 2 Group Water and Environment, SINTEF Building and Infrastructure, Richard Birkelands vei 3, \\ 7034 Trondheim, Norway; Gema.Raspati@sintef.no (G.S.R.); Rita.Ugarelli@sintef.no (R.M.U.) \\ 3 Department of Civil, Environmental and Mechanical Engineering, University of Trento, 38123 Trent, Italy; \\ Paolo.Bertola@unitn.it \\ 4 Department of Civil and Environmental Engineering, Norwegian University of Science and Technology, \\ S.P. Andersens veg 5, 7491 Trondheim, Norway \\ * Correspondence: marianna_dercole@hotmail.it; Tel.: +39-320-042-1938
}

Received: 17 December 2017; Accepted: 1 March 2018; Published: 6 March 2018

\begin{abstract}
The efficient and effective management of existing water distribution systems (WDSs) faces challenges related to aging of infrastructure, population growth, extended urbanization, climate change impacts and environmental pollution. Therefore, there is a need for integrated solutions that support decision makers to plan today potential interventions, considering the possible consequences and variations in mid- and long-term perspectives. This study is a part of a more comprehensive project, where advanced hydraulic analysis of WDS is coupled with a dynamic resources input-output analysis model. The proposed modeling solution provides a robust tool to support planning of intervention actions and can be applied to optimize the performance of a water supply system considering energy consumption and environmental impacts. This paper presents an application of the proposed method in pipe rehabilitation/replacement planning, maximizing the network mechanical reliability and minimizing the risk of unsupplied water demand and pressure deficit evaluated at nodal level, under given economic constraints.
\end{abstract}

Keywords: water distribution; management; mechanical reliability; risk assessment

\section{Introduction}

According to an Environmental Protection Agency (EPA) report [1]: "System rehabilitation is the application of infrastructure repair, renewal, and replacement technologies to return functionality to a drinking water distribution system or a wastewater collection system". The process of intervention planning and prioritization is a function of a network's current condition assessment, the extent of critical repair needs, the availability of funding for rehabilitation work options, and the ability to inspect and assess the condition and deterioration rate of each element [2]. Asset management activity and life cycle analysis drive the broad activities that determine system-wide planning.

Among the possible alternatives for leakage reduction, asset replacement is quite expensive compared to active leakage control (ALC) and pressure management (PM). However, if the condition of the underground assets is so poor ALC and PM do not provide a sustainable solution. A well-managed water loss program should always include a budget for selective replacement of mains and/or service pipes specifically to reduce leakage if ALC, or PM is no longer a feasible option to mend the situation [3]. Knowing when, where and how to rehabilitate pipes requires a good knowledge of the system 
performance, its conditions and the availability of decision support systems for rehabilitation planning. The present study describes a replacement planning approach based on mechanical reliability to minimize unsupplied water demand and pressure deficit.

\section{Reliability Theory Applied to Water Distribution System}

The definition of reliability is not unique, but depends on the specific field in which it is applied. Therefore, it is more precise to use this term in a general sense to indicate the overall ability of a system to perform its function [4]. The mechanical and electrical complex systems are the main sectors where the theory of reliability found the initial application and only later was applied to hydraulic systems that exhibit some analogues aspects with those of the production, transport and distribution. Reliability is commonly defined, among other definitions, as "the probability of a device performing its purpose adequately for the period of time intended under the operating conditions encountered" [5]. This comprises the concept of probability, adequate performance, time and operating conditions [6].

For water distribution systems (WDSs), several types of reliability can be defined, in theory one for each set expected function of an asset or of the entire network [7]. However, the literature has mainly focused on the concepts of mechanical and hydraulic reliability. The mechanical reliability can be defined as the probability that a component (new or repaired) experiences no structural failures during the time interval from time zero to time $t(0, t)$. The hydraulic reliability refers to the probability that a water distribution pipe can meet a required water flow level at a required pressure at each nodal demand [8]. Walski [9] observed that the topic of reliability is integrated to all parts of decision related to WDS design, operation and maintenance, even though most evaluations of reliability tend to focus on the design of the system. If the WDS has a sufficient redundancy to deliver water and is able to perform the expected function even for an aging infrastructure, it is therefore considered reliable. Moreover Kanakoudis et al. [10,11] observed that reliability is the most common performance indicators used as maintenance priority criterion.

The reliability analysis could be used to identify repair works on existing system [12] considering various random factors such as customer demand, mechanical failures, roughness indices, that could affect the performance or in the expansion of existing networks [13] where the reliability is maximized with the support of computer models. Among the existing models to analyze the reliability of a water distribution system, "Management module" in WDNetXL (Version 4.0, IDEA RT, http:/ / www. hydroinformatics.it) $[14,15]$ is a tool that enables reliability analysis of the network by three specific functions: Reliability One Failure, Reliability Multiple Failure and Hydraulic Reliability. The first two functions analyze hydraulic behavior of a WDS by simulating single or multiple pipe or node failures/disconnections. Hydraulic Reliability function performs the analysis of the network hydraulic behavior by varying the boundary conditions such as pipe hydraulic resistances, background leakages, nodal customer demands, nodal free-orifice demands, and their combinations.

Reliability One-Failure function, which was used in this study, investigates all failure scenarios generated by disconnection of single pipe or node from the network. Given that a link may represent not only a pipe but also a device (valve or pump), a pipe failure can be associated also as a device failure. The reliability indicators proposed in this study are based on two parameters: unsupplied demand and pressure deficit. Both parameters are assessed from the failure events considered in Reliability One Failure function in a pressure driven, extended period simulation [16] and are associated with Isolation Valve system (IVS) that disconnects the failing pipe or node from the rest of the network [17,18]. Therefore, the study of WDS behavior resulting from failure events can be considered a mechanical reliability analysis.

The break rate $\lambda$ that represents the number of break per kilometer per year is a common parameter associated with the mechanical reliability analysis [19]. It is dependent on many factors such as installation year, pipe corrosion, diameter, break type, pipe material, seasonal variation, soil environment, break history, pressure, land use and pipe length. Consequently, to consider these factors individually to obtain a prevision of the expected break rate is a rather difficult task. Break rate is 
case specific and, therefore, it is advisable to calculate it at a cohort level through an analysis of the historical break data related to the specific network. Otherwise, it is also possible to use formulae taken from the literature from a similar case study. The break rate is assessed for different pipe cohorts defined by similar characteristics and grouped to have a representative statistical sample. Afterwards, the specific number of breaks per year is evaluated for each pipe by multiplying $\lambda$ with the individual pipe length.

\section{Methodology}

Figure 1 depicts the proposed reliability-based risk assessment methodology. The analysis starts with the creation of the hydraulic model for the specific case study in WDNetXL environment $[14,15]$. Mechanical reliability is done by running simulations in "Reliability One Failure" function that evaluate the hydraulic behavior in term of unsupplied demand and pressure deficit after each failure event. The specific contribution of this study is to couple failure statistics with a risk based ranking of pipes for rehabilitation by using the reliability indicators calculated from WDNetXL simulation results.

\begin{tabular}{|c|c|c|c|c|c|c|c|}
\hline \multicolumn{8}{|c|}{ Starting condition } \\
\hline 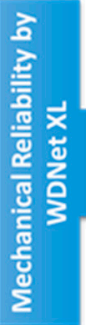 & $\begin{array}{l}\text { - Mechanical reliability } \\
\text { analysis of the network } \\
\text { - Calculation of unsupplied } \\
\text { demand and pressure } \\
\text { deficiency due to failure on } \\
\text { pipe segment level }\end{array}$ & 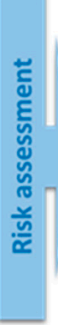 & $\begin{array}{l}\text { Calculation of } \\
\text { consequence, risk } \\
\text { probability and } \\
\text { overall risk } \\
\text { valuation on } \\
\text { individual pipe } \\
\text { level }\end{array}$ & 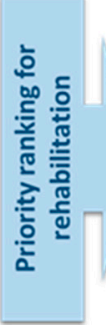 & $\begin{array}{l}\text { Definition } \\
\text { of criteria } \\
\text { for risk } \\
\text { ranking }\end{array}$ & 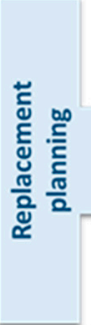 & $\begin{array}{l}\text { Overall risk } \\
\text { reduction } \\
\text { evaluated based } \\
\text { on rehabilitation } \\
\text { target in } \\
\text { masterplan } \\
\text { - Project planning, } \\
\text { execution, } \\
\text { evaluation, etc. }\end{array}$ \\
\hline
\end{tabular}

Figure 1. Methodology of reliability-based risk assessment proposed by this study.

\subsection{The Reliability Indicators Implemented in WDNetXL}

Many WDS performance indicators found in the literature were initially developed by agencies such as International Water Association (IWA), the American Water Works Association (AWWA), Asian Development Bank, (ADB), National Research Councils (NRCs), National Water Commissions (NWCs), World Bank (WB). Such indicators were then studied, improved and implemented [20-24].

Several authors $[12,25,26]$ evaluated reliability by using performance indicators on the basis of the ratio between volumes actually delivered during the evaluation period and the volume required at a given node. A performance indicator relative to pressure that considers the ratio between the minimum pressure value and pressure required was also proposed [27].

WDNetXL model evaluates in extended period simulation (EPS) unsupplied customer demand $(U N)$ and pressure deficit $(P R)$ evaluated at node level after each failure event. These two parameters represent the actual values of customer demand and pressure after a failure event occurs and are compared with those in a normal condition, that is, condition in which no failure occurs. UN and $P R$ are defined as [28]:

$$
\begin{aligned}
& U N_{i, e, t}=1-\frac{d_{i, e, t}^{a c t}}{d_{i, 0, t}^{r e q u}} i \epsilon n_{n} \text { eє } n_{e} t \in[1, T] \\
& P R_{i, e, t}=1-\frac{p_{i, e, t}^{a c t}}{p_{i, 0, t}^{\text {normal }}} \quad i \in n_{n} \text { ee } n_{e} t \in[1, T]
\end{aligned}
$$

where: 
- $\quad i, e$ and $t$ are subscripts indicating respectively the $i$-th node, the $e$-th failure event and the time $t$ of the EPS during time interval $T ; e=0$ represents for normal condition;

- $\quad d^{\text {act }}$ and $p^{a c t}$ are the actual customer demand computed in pressure driven analysis (PDA) using the Wagner's model [29] and actual nodal pressure evaluated in PDA or demand driven analysis (DDA).

- $d^{r e q u}$ is the required customer demand varying over time;

- $\quad p^{\text {normal }}$ is the nodal pressure in normal conditions computed varying over time;

- $n_{n}$ and $n_{e}$ are the number of nodes and events, respectively.

It is clear that in normal condition $d^{\text {act }}$ and $p^{a c t}$ are equal or close to $d^{\text {requ }}$ and $p^{\text {normal }}$. Thus, the corresponding fractions $\frac{d^{a c t}}{d^{\text {requ }}}$ and $\frac{p^{a c t}}{p^{\text {normal }}}$ are close to unity, given the indicators $U N$ and $P R$ are equal to or close to zero. This means that there is small or no deficiency between the supplied and required values and the condition of the $i$-th node are 'good'. If a failure is imposed, the values of $d^{a c t}$ and $p^{a c t}$ are no longer equal or close to $d^{\text {requ }}$ and $p^{\text {normal }}$. Thus, $\frac{d^{\text {act }}}{d^{\text {requ }}}$ and $\frac{p^{\text {act }}}{p^{\text {normal }}}$ give a value that are less than unity. This means there is a deficiency between the supplied and required values. Therefore, $U N$ and $P R$ values would be larger than zero. The larger values of $U N$ and $P R$ represent the worse condition of unsupplied demand and pressure deficit. Note that for isolated nodes, for example, due to valve shutdowns, $d^{a c t}$ and $p^{a c t}$ are null and the corresponding fraction $U N$ and $P R$ are unitary.

Based on $U N$ and $P R$ evaluated by WDNetXL, the results are then elaborated in a risk analysis for the nodes in the network affected by each failure event. This enables classification of pipes based on the risks they impose to the performance of WDS should a failure occurs to them. The EPS is done on an hourly basis for the 24-h simulation period. Considering each node for the specific failure event, the maximum value that represents the worse condition for that node in the day is registered. A value of $U N$ and $P R \geq 0.5$ is chosen to define a critical situation in which the demand and pressure are less than the $50 \%$ of the normal condition. This value is arbitrary and can be chosen specifically by the decision makers. Figure 2 depicts a visualization of critical nodes after each failure event. At this stage, the methodology ranks failure events in term of the number of affected nodes from two indicators, $U N$ and $P R$.

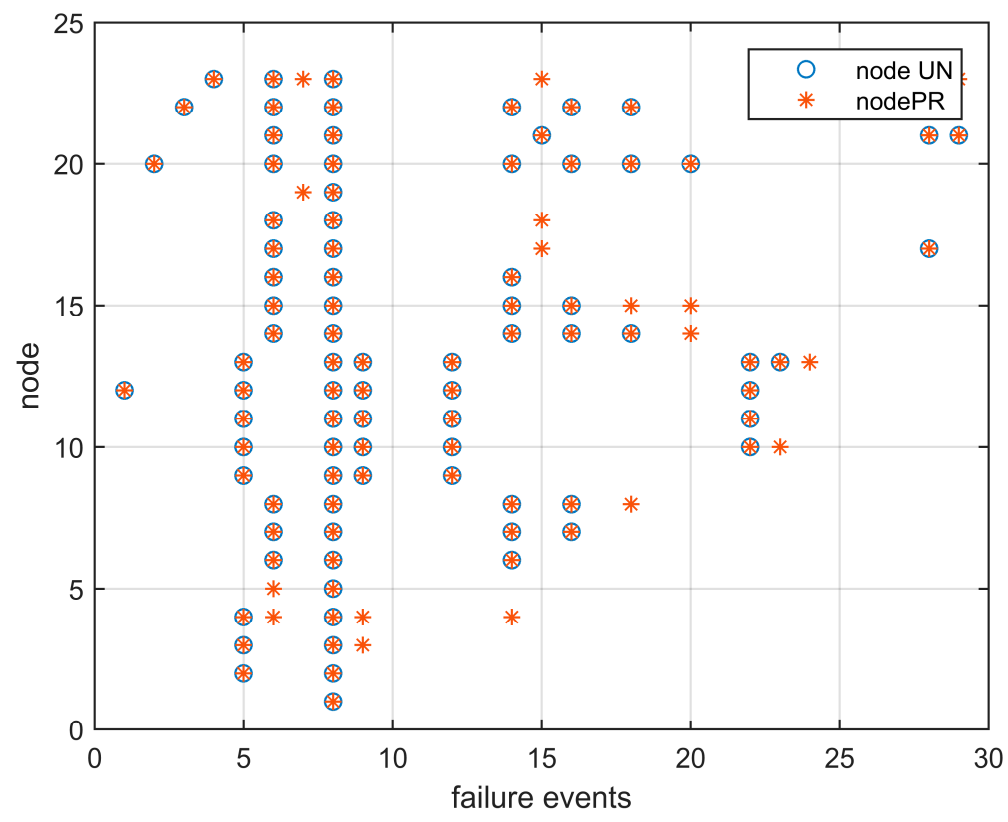

Figure 2. Example of a scatter plot of critical nodes that are affected by each failure event identified by node ID. 
The use of IVS helps isolate parts of the network creating segments, that is, the smaller portions of a distribution system. By performing network segmentation, one can assume that a failure event related to a specific pipe segment deals with all pipes belonging to the same pipe segment and, therefore, the same hydraulic importance is assigned to these pipes belonging to that specific pipe segment.

\subsection{Risk Assessment Approach}

Risk methodologies are intended to evaluate risks associated with the existing system and possible intervention options, and to contribute to the understanding of how decisions can contribute to meeting performance targets. Multicriteria decision analysis (MCDA) methods need to be used for aggregation and ranking tasks. Decision criteria can be used in parallel metrics of risk as well as of performance and cost. Risk values, crossing probability scenario and their consequences, will be passed to the MCDA to provide a possible ranking for competing alternatives.

There are many definitions of risk and risk management. ISO 31000:2009-Risk Management [30] and ISO Guide 73:2009-Risk Management-Vocabulary [31], define risk as the "effect of uncertainty on objectives". ISO Guide 73 also states that an effect may be positive, negative or a deviation from the expected. The risk is often described by an event related to a change in circumstances or a consequence and by the associated likelihood of occurrence. It is important to underline that the term likelihood refers to the probability of an event occur. Such a probability can be defined, measured or determined objectively or subjectively, and is described using general terms or mathematically (such as a probability or a frequency over a given time period). For example, Kanakoudis [11] associated the probability of a failure with the magnitude of the failure impacts in the Significance Index. In this study, the risk assessment is performed by combining the probability for each pipe to break with the consequence induced in terms of $U N$ and $P R$. Therefore, the risk associated to $p$-th pipe after a failure event is defined as:

$$
\text { risk }_{p}=\lambda \times C_{\text {tot }_{p}}=\text { frequency } \times \text { consequence }
$$

where:

- $\lambda$ represents the frequency of break in a year;

- $\quad C_{\text {tot }_{p}}$ is the overall consequence $p=C_{p}^{U N \text { dem }} \times C_{p}^{P R}$;

- $\quad C_{p}^{U N \text { dem }}$ is the consequence in term of $U N_{\text {dem }}=$ break rate $_{\text {norm }} p \times n_{\text {critical nodes } U N \text {; }}$;

- $\quad C_{p}^{P R}$ is the consequence in term of $P R=$ break rate $_{\text {norm }} \mathrm{p} \times n_{\text {critical nodes } P R}$;

- $\quad p$ subscript indicating the $p$-th pipe.

The combined probability and consequence for each pipe for all failure events is depicted in a risk matrix as in Figure 3. Figure 4 is another example in which the pipes are ranked according to risk value sorted in descending order. The visualizations help support decision makers to decide the risk reduction measure to adopt should be more preventive (reduce the probability for the event to happen) or protective (mitigate consequences). 


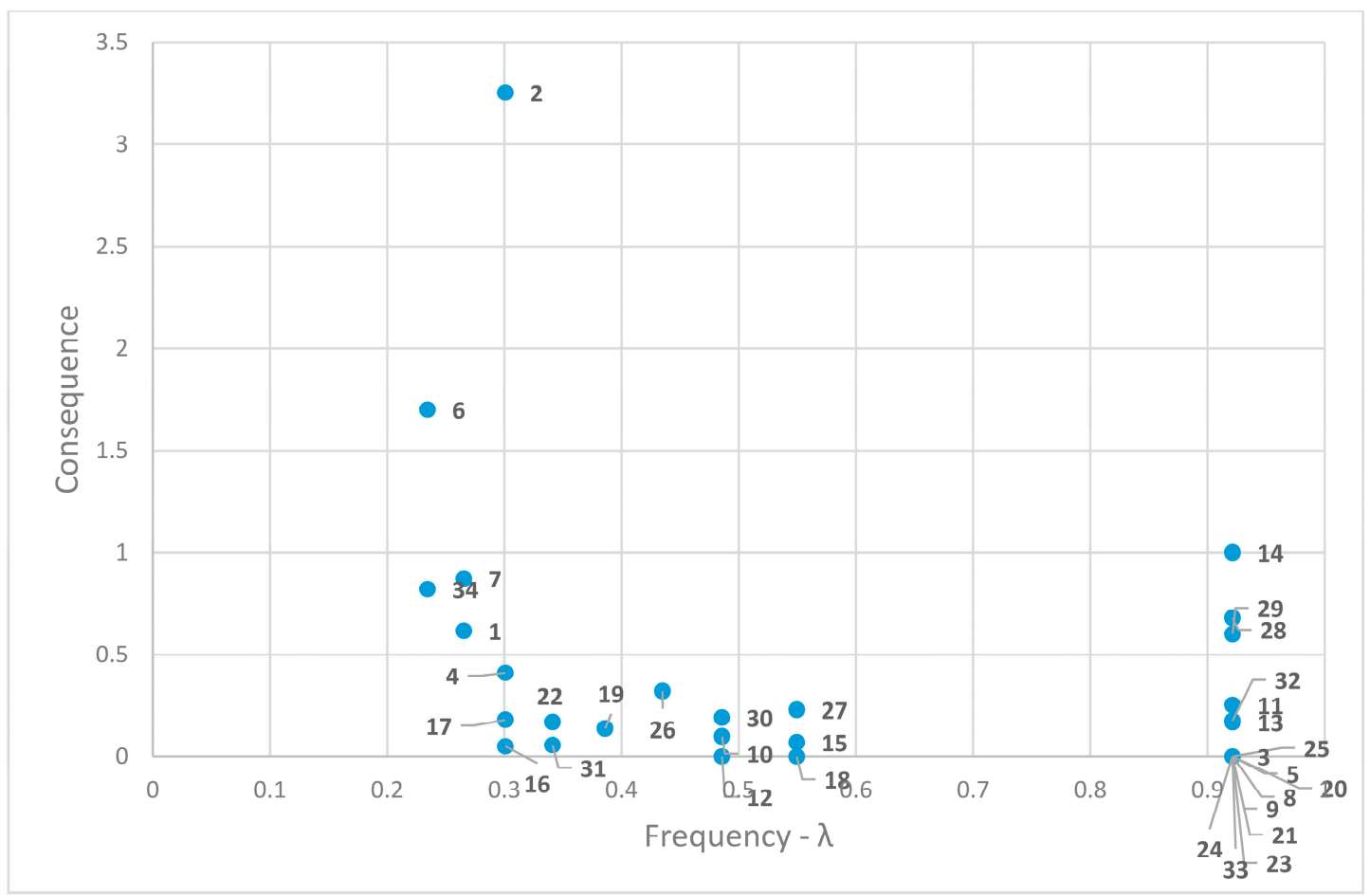

Figure 3. Frequency of break and relative consequence.

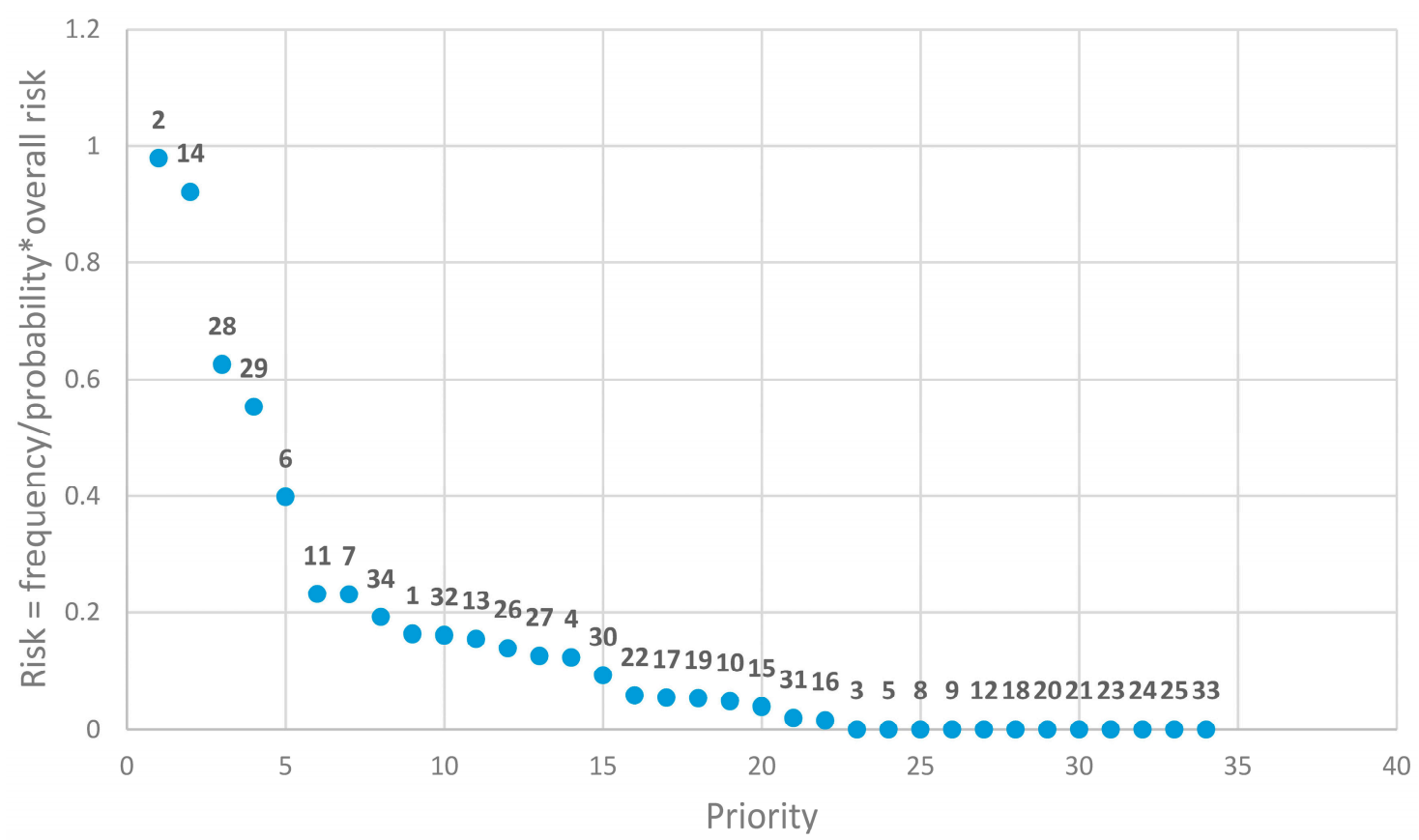

Figure 4. Risk values sorted in descending order.

\subsection{Replacement Planning}

The prioritization and selection of the intervention options require the adoption of a MCDA methodology. The objective is to minimize the residual risk, after rehabilitation of a given pipe to ensure maximum reliability for example for a given annual investment. The proposed methodology 
ranks pipes with the objective to minimize residual risk and maximize reliability to meet the available budget. The cumulative direct cost is defined as:

$$
\operatorname{cum}_{\operatorname{cost}}=\operatorname{cost}_{p}+\sum_{k=1}^{p-1} \operatorname{cum}_{\operatorname{cost}} \text {. }
$$

where:

- $\quad p$ depicts the $p$-th pipe

- $\operatorname{cost}_{p}$ is the direct cost of the $p$-th pipe

- $\quad \sum_{k=1}^{p-1}$ cum $\cos t_{k}$ is the cumulative sum of the direct costs of pipes until pipe $p-1$

The cumulative risk reduction, cum risk reduction $_{p}$, is evaluated considering the risk sorted in descending order:

$$
\text { cum risk reduction }_{p}=\text { risk }_{p}+\sum_{k=1}^{p-1} \text { cum risk }_{k} .
$$

where:

- risk $_{p}$ is the risk associated to the $p$-th pipe

- $\quad \sum_{k=1}^{p-1}$ cum risk reduction $_{k}$ is the cumulative sum of the risks of pipes until pipe $p-1$

The residual risk is evaluated as:

$$
\text { residual risk } k_{p}=\max (\text { risk reduction })-\text { cum risk reduction } p_{p}
$$

Given a fixed annual investment budget for each intervention of rehabilitation, the remaining budget is assessed and used to fit further interventions minimizing the actual residual risk and provide maximum service. Figure 5 shows an example of the analysis of cumulative risk reduction evaluated based on a specific budget. The cumulative residual risk curve is based on the pipe ranking expressed in cumulative pipe cost. The red line represents cumulative risk reduced up to the point where the direct cost of pipe replacement is covered by the investment budget (grey line).

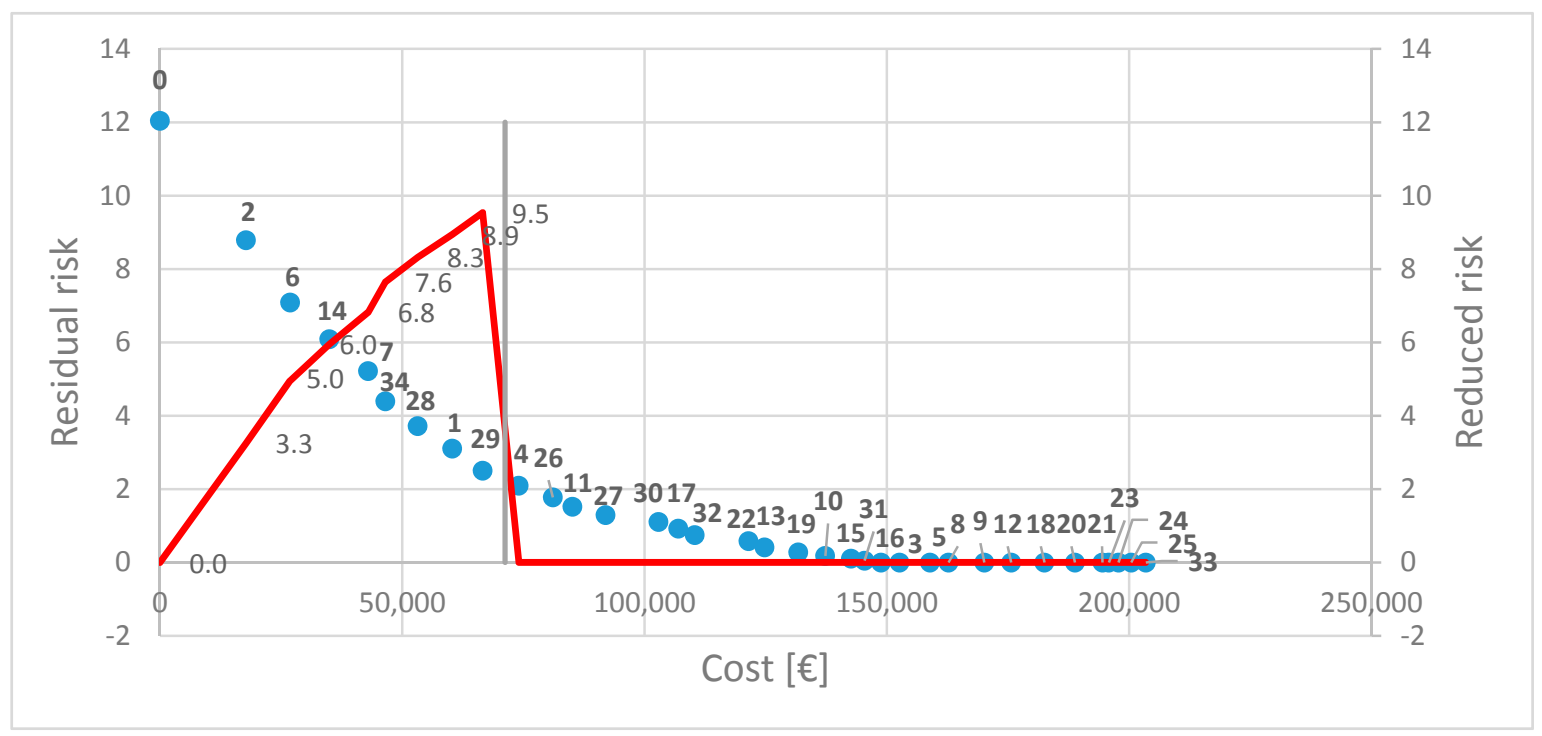

Figure 5. Example of cumulative risk reduction evaluated based on a specific available budget. 
The cumulative residual risk value peaks at cumulative cost equal to zero, representing the total risk if no replacement program is implemented. The cumulative risk starts decreasing if the replacement program is executed and this is limited by the available budget or for example by a target length of pipe replacement/rehabilitation. In theory, the cumulative risk is equal to zero if all pipes in the network are replaced.

\section{Case Study-Laives}

The methodology described above has been applied to the Laives water distribution network, a town in the province of Bolzano, Italy. It serves about 18,000 customers and spreads from the districts of San Giacomo in the south of Bolzano until the industrial area of Laives (Figure 6). The three interconnected districts subdivide the network are: San Giacomo, Pineta and Laives. Each of these districts has a tank supplied by wells or springs.

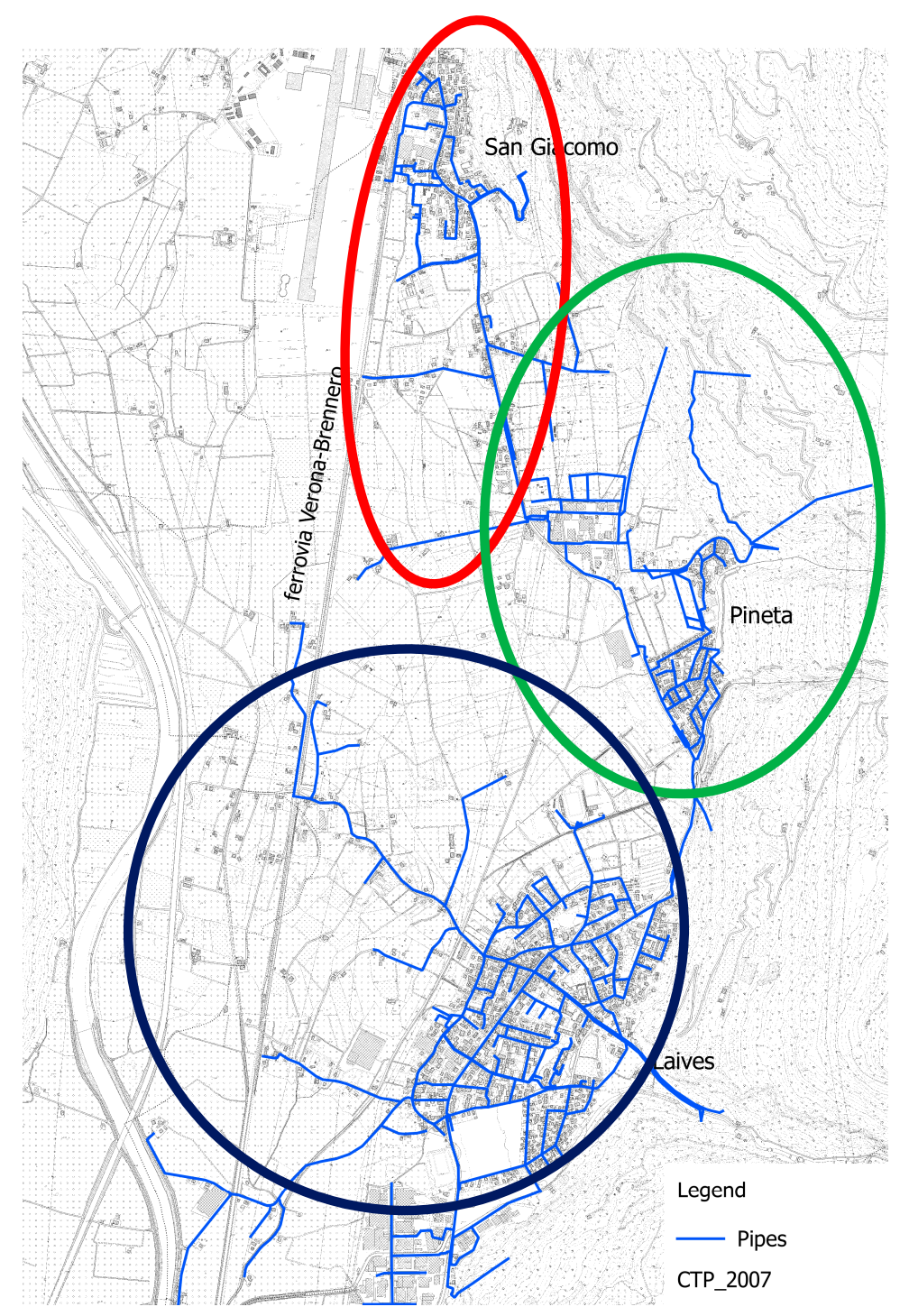

Figure 6. Geographical representation of Laives water distribution network and the three interconnected districts of San Giacomo, Pineta and Laives.

Pipe materials that characterize the network are ductile iron and mild steel that cover more less $58 \%$ and $37 \%$ of the network, respectively. Other pipe materials, such as Polyethylene (PE) (4\%) and Polyvinylchloride (PVC) (1\%) are also installed. The Laives network is modelled using WDNetXL 
and is characterized by 375 nodes and 439 pipes of which 18 are valves and pumps. Some of the pumps present in the network work with different regimes during the hours of the day to save energy. To simulate this, parallel pumps are added working in a different range of hours and with distinct levels in the tanks.

In this paper, the mechanical reliability analysis considered in WDNetXL "Management module" is the 'Pipe Failure' type that evaluates the impacts of closure of a particular isolation valve system/pipe segment to UN and PR. The mechanical reliability simulation considering an 'N-rule' valve system that assumes two valves for each pipe trunk and results in 379 failure events; each corresponds to a specific segment in the Laives Network.

\section{Results and Discussion}

The scatter plot of nodes that are affected by each failure event is reported in Figure 7. Some of the nodes exhibit unsupplied demand (UN), pressure deficiency $(P R)$, or both conditions simultaneously. The results of the first simulation show that the most part of the worst condition registered at nodes are of $P R$, rather than of $U N$. This is because $P R$ takes a reference value of the nodal pressure in the normal condition $\left(p^{\text {normal }}\right)$ that for the Laives network is characterized by a generalized high level of pressure distribution, much higher than 30-40 m. Therefore, a new simulation was performed by lowering the reference value down to the minimum pressure level for users defined by regulation of the province of Bolzano, which is of $40 \mathrm{~m}$ water column (see Figure 7). In the following, the $P R$ indicator is presented with subscript "ser" to indicate the reference value to service pressure (PRser).

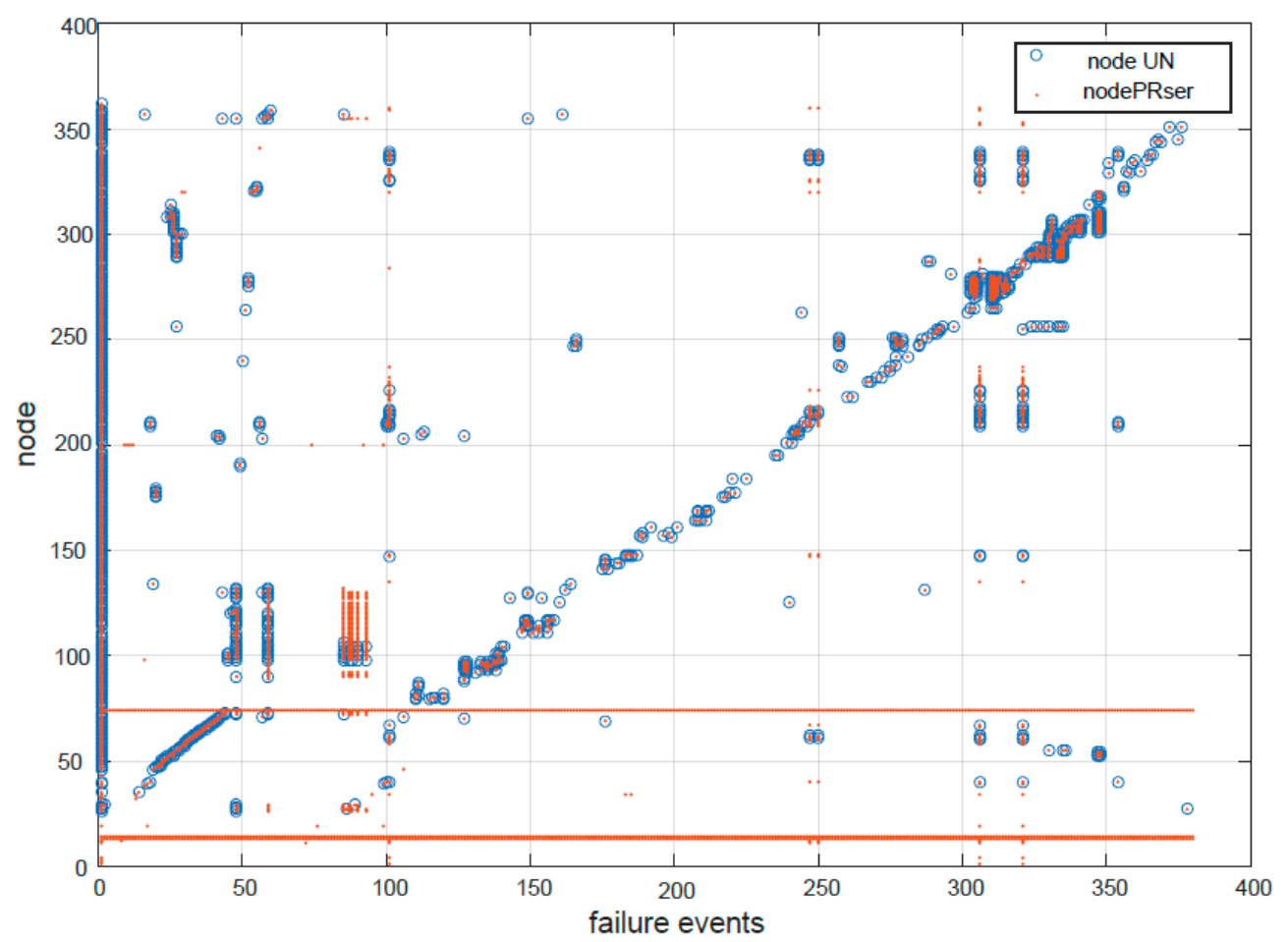

Figure 7. Scatter plot of node IDs that are affected by each failure event in case study of Laives.

Table 1 reports the top 10 events ranked based on the number of affected nodes from the simulation and the location of the five common failure events in the network is shown in Figure 8. It is interesting to observe the different ranks of failure events that lead to highest number of nodes affected by $U N$ and PR. In addition, for example the last five failure events causing $U N$ are absent on the list of failure events causing $P R$. 
Table 1. List of top-10 failure events corresponding to the number of affected nodes.

\begin{tabular}{ccccc}
\hline \multicolumn{2}{c}{ UN } & \multicolumn{2}{c}{ PRser } \\
\hline Rank & Failure Event & Number of Affected Nodes & Failure Event & Number of Affected Nodes \\
\hline 1 & 48 & 34 & 306 & 54 \\
2 & 59 & 29 & 321 & 54 \\
3 & 321 & 29 & 59 & 45 \\
4 & 306 & 28 & 101 & 42 \\
5 & 101 & 20 & 85 & 40 \\
6 & 347 & 16 & 87 & 37 \\
7 & 348 & 16 & 90 & 37 \\
8 & 335 & 14 & 88 & 35 \\
9 & 26 & 13 & 93 & 35 \\
10 & 310 & 13 & 48 & 34 \\
\hline
\end{tabular}

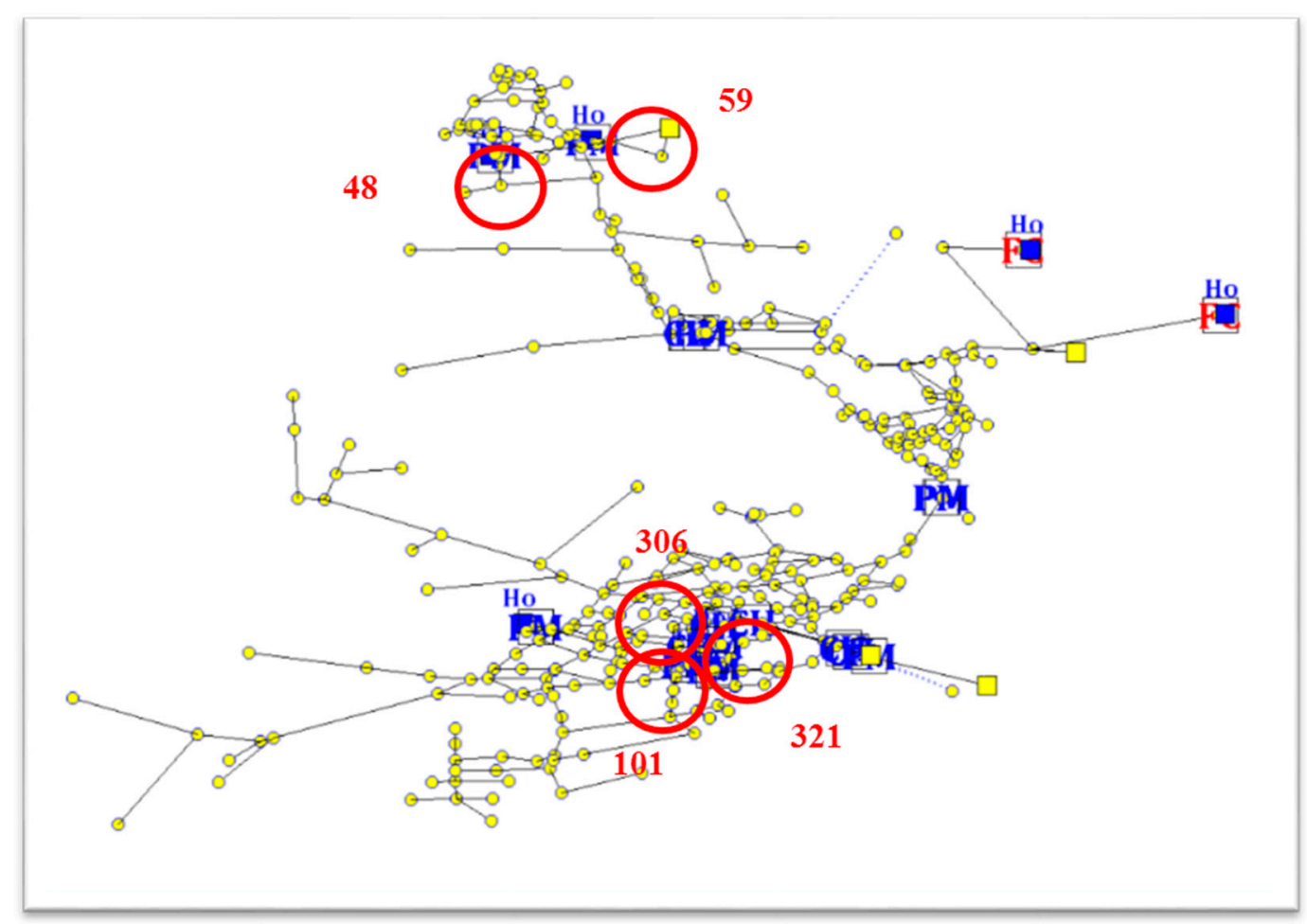

Figure 8. Locations of the five common failure events in Laives water distribution network.

Table 2 shows an extract of risk calculation for some pipes of the network. One can observe that the number of affected nodes with $U N$ are lower than that with PR. Consequently, the consequence in term of unsupplied demand, $\mathrm{C}_{\mathrm{p}}$ UNdem and consequence in term of pressure deficit, $\mathrm{C}_{\mathrm{p}}{ }^{\text {PRser }}$ are affected by these differences.

Table 2. An extract of risk calculation based on Equation (3).

\begin{tabular}{cccccccccccc}
\hline Failure Event & Pipe ID & Length & UN & PRser & $\boldsymbol{\lambda}$ & Break & Break Norm & $\mathbf{C}_{\mathbf{p}}$ UNdem & $\mathbf{C}_{\mathbf{p}}$ PRser & C $\_$tot $^{\text {Risk }}$ \\
\hline 87 & 32 & 41.62 & 4 & 37 & 0.601 & 0.025 & 0.028 & 0.112 & 1.035 & 0.116 & 0.070 \\
88 & 33 & 53.37 & 2 & 35 & 0.601 & 0.032 & 0.036 & 0.072 & 1.255 & 0.090 & 0.054 \\
90 & 34 & 19.36 & 4 & 37 & 0.601 & 0.012 & 0.013 & 0.052 & 0.482 & 0.025 & 0.015 \\
93 & 35 & 28.63 & 2 & 35 & 0.601 & 0.017 & 0.019 & 0.038 & 0.674 & 0.026 & 0.016 \\
\hline
\end{tabular}

Figure 9 shows the ranking of portion of pipes in term of risk (the first twenty pipes exhibiting highest risk), the corresponding risk values are reported in Table 3 , with the relative values of $\lambda$ and 
$\mathrm{C}_{\text {tot }}$. By comparing the list of failure events in Table 1, with the list of the first twenty pipes ranked following the risk evaluation in Table 3, only three of the predominant failure events in Table 1 appear in the ranking list. This is due to the effect of $\lambda$ acting as a weight ranking.

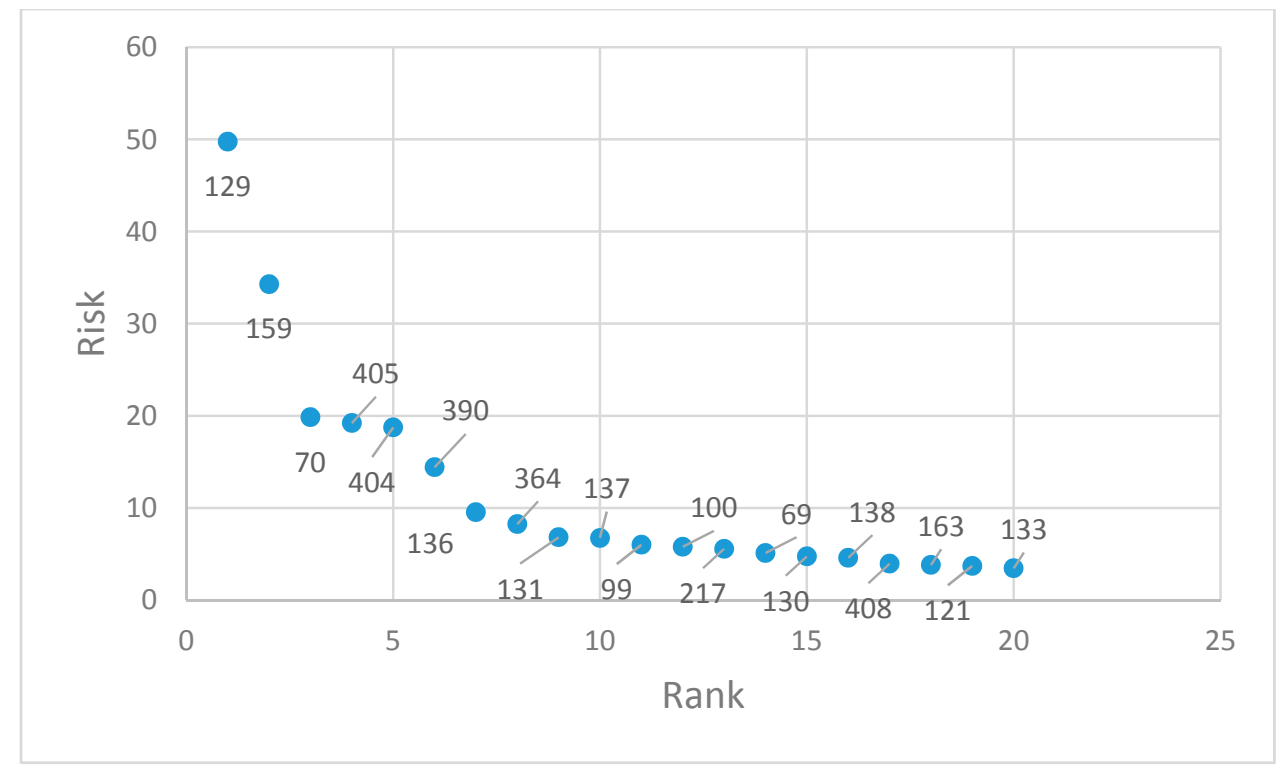

Figure 9. Priority ranking of the pipes with highest calculated risk values.

Table 3. Ranking list of the pipes with highest risk values and the corresponding failure event number.

\begin{tabular}{cccccc}
\hline Rank & Failure Event & Pipe ID & $\lambda$ & $\mathbf{C}_{\text {tot }}$ & Risk \\
\hline 1 & 312 & 129 & 1.164698 & 42.71896815 & 49.75472 \\
2 & 328 & 159 & 1.164698 & 29.4251172 & 34.27139 \\
3 & 27 & 70 & 0.921421 & 21.52369527 & 19.83238 \\
4 & 59 & 405 & 0.601952 & 31.91113549 & 19.20899 \\
5 & 59 & 404 & 0.601952 & 31.12305147 & 18.7346 \\
6 & 48 & 390 & 0.728958 & 19.76419825 & 14.40727 \\
7 & 52 & 136 & 1.164698 & 8.184900227 & 9.532941 \\
8 & 135 & 364 & 1.164698 & 7.065763846 & 8.229484 \\
9 & 305 & 131 & 0.728958 & 9.332392247 & 6.802922 \\
10 & 52 & 137 & 1.496754 & 4.497545577 & 6.731719 \\
11 & 42 & 99 & 1.164698 & 5.14500632 & 5.992381 \\
12 & 42 & 100 & 1.164698 & 4.966474288 & 5.784445 \\
13 & 247 & 217 & 0.921421 & 6.021586638 & 5.548415 \\
14 & 27 & 69 & 0.921421 & 5.53121004 & 5.096572 \\
15 & 304 & 130 & 1.164698 & 4.058647449 & 4.7271 \\
16 & 313 & 138 & 0.921421 & 4.96502344 & 4.574876 \\
17 & 48 & 408 & 0.728958 & 5.395299548 & 3.932946 \\
18 & 27 & 163 & 1.164698 & 3.258599588 & 3.795286 \\
19 & 321 & 121 & 0.728958 & 5.067614029 & 3.694078 \\
20 & 315 & 133 & 1.164698 & 2.94751094 & 3.432961 \\
\hline
\end{tabular}

Figure 10 shows the cumulative risk calculated for all pipes in the network. By replacing particular pipes in Table 3 will lead to reduction of risk proportional to the risk imposed by the pipes. The cumulative value peak at zero represents the total risk if no replacement program is implemented. 


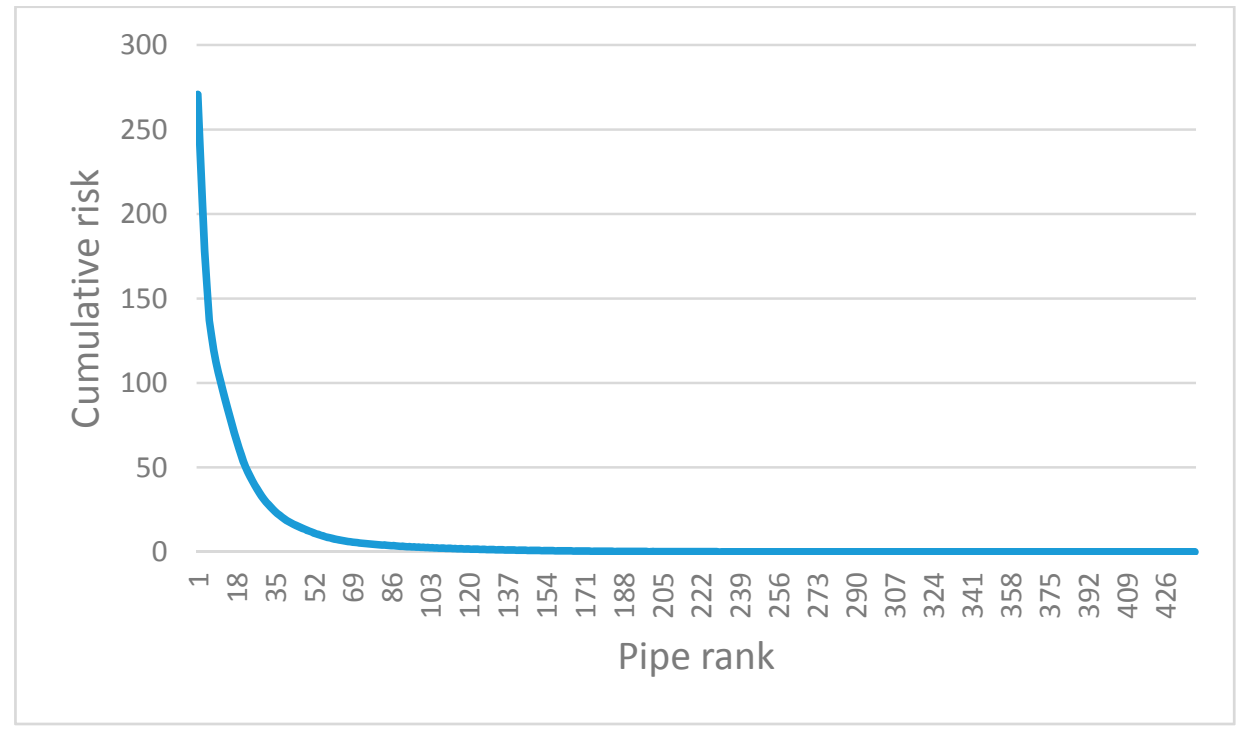

Figure 10. Cumulative risk calculated based on pipe ranking.

The ranking of pipes could be driven by other constraints than cost, as for instance the requirement set by regional authorities on rehabilitation targets to be met. In the case of Laives, the target replacement rate is $2.5 \%$ of pipe network/year [32], which corresponds to substitution of around 10 pipes a year.

Considering this alternative constraint, an additional analysis was performed and the results are presented in Figure 11. The $x$-axis is a blow up of $x$-axis in Figure 10. This section is meant to put emphasis on its potential application in the replacement-planning phase with respect to the risk asset management principle discussed in previous section. The orange line represents cumulative risk reduction up to the point where the number of pipes to replace is equal to 10. Following the replacement program, as seen from the graph, the cumulative risk reduces by almost $66 \%$ from 313.41 to 106.34 (corresponding to cumulative risk reduction of 207.07).

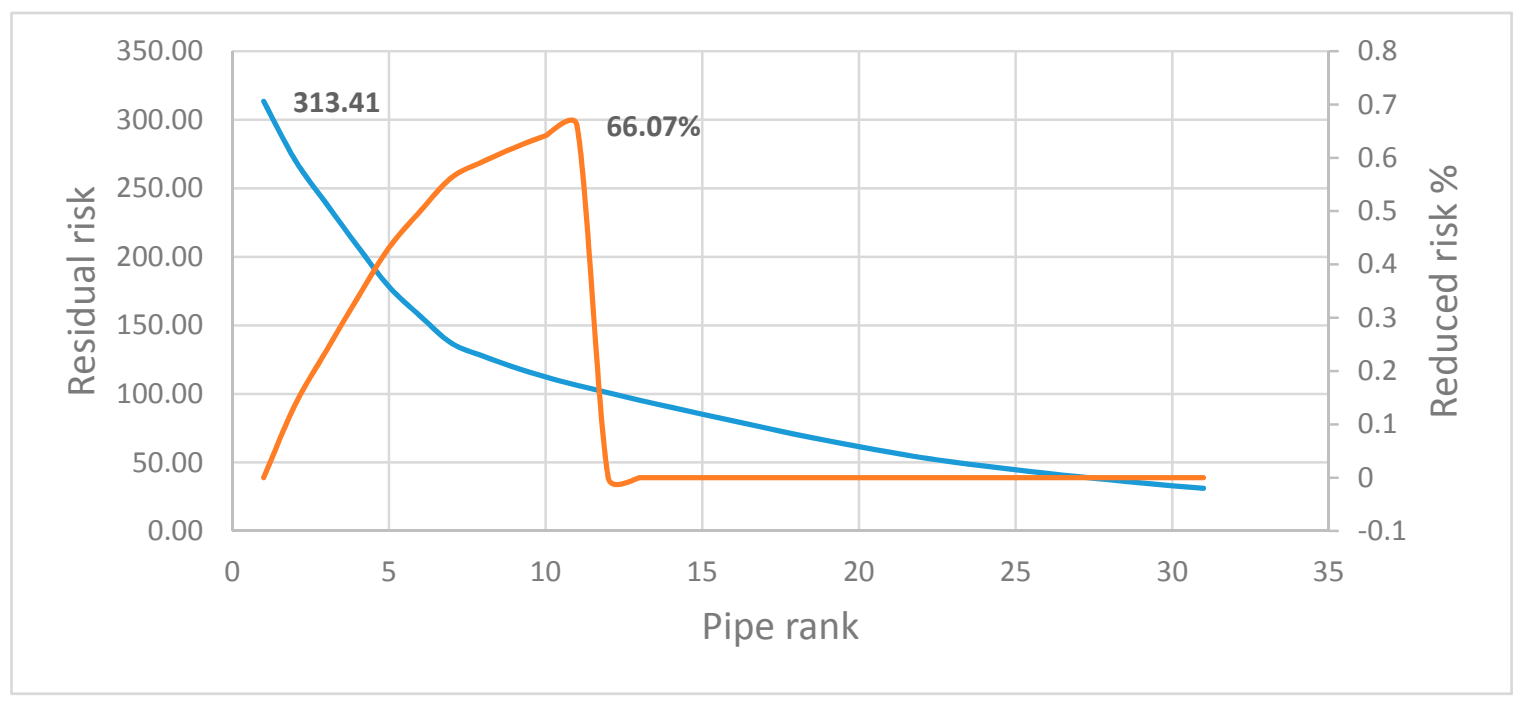

Figure 11. Cumulative risk reduction evaluated based on the target replacement percentage of pipe in the masterplan calculated for the first year. 


\section{Conclusions}

Many factors, such as aging of infrastructure, population growth, increasing urbanization, and including more recent factors such as climate change and environmental pollution, require a change in the management of the WDS. This paper, part of a more comprehensive project of optimization of the performance of a water supply system, presents an application of mechanical reliability analysis in WDNetXL in pipe rehabilitation/replacement planning. The inclusion of statistical information of pipe break rate allows risk assessment at an individual pipe level that can be used to develop a priority ranking for pipe replacement. The proposed method is applied to the network of Laives, a town in province of Bolzano. The effect of the break rate $(\lambda)$ as the ranking weight is highlighted in this study. This approach can also be extended to evaluate the risk reduction reached once the replacement plan is executed.

Data availability for break rate calculation has been a limiting factor in this study. To develop a good pipe replacement plan, the real break rate with a minimum of 10-year pipe break history should be considered. Consequently, the 'map' of the pipes that need replacement must be updated owing to the change of break rate once a pipe rehabilitation/replacement is commenced. At the same time, inclusion of a break rate that considers also the pipe age and materials, not only pipe diameter as in this study, will be more useful in representing the actual condition of the network. The ongoing research considers the age factor and its influence on a long-term planning by observing the dynamics of pipe-risk ranking over time.

For each replacement, the main constraint considered was the direct cost, but it is possible to evaluate the energy consumption and the $\mathrm{CO}_{2}$ emissions connected using the integrated modeling approach as will be addressed later by this study, that is, by incorporating MCDA.

Acknowledgments: This work has been partially carried out within the Research project "AI-ALPEN", CUP: B26J16000300003 funded by the PAB (Autonomy Provence of Bozen-Italy) for University Research-2014. The EFRE-FESR project "Thermo Fluid Dynamics, infrastructures for applied research" (ERDF 2014-2020, CUP: I52F16000850005) support is also acknowledged.

Author Contributions: Maurizio Righetti, Paolo Bertola and Rita Maria Ugarelli have supervised this study. Marianna D'Ercole and Gema Sakti Raspati have contributed for data analysis and manuscript preparation.

Conflicts of Interest: The authors declare no conflict of interest.

\section{References}

1. Environmental Protection Agency (EPA). Innovation and Research for Water Infrastructure for the 21st Century-Research Plan; U.S. Environmental Protection Agency, Office of Research and Development-National Risk Management Research Laboratory: Cincinnati, OH, USA, 2007.

2. Environmental Protection Agency (EPA). Rehabilitation of Wastewater Collection and Water Distribution Systems-State of Technology Review Report; U.S. Environmental Protection Agency, Office of Research and Development-National Risk Management Research Laboratory: Cincinnati, OH, USA, 2009.

3. European Commission. EU Reference document Good Practices on Leakage Management WFD CIS WG PoMMain Report; European Commission: Brussels, Belgium, 2015.

4. Billinton, R.; Li, W. Basic Concepts of Power System Reliability Evaluation. In Reliability Assessment of Electric Power Systems Using Monte Carlo Methods; Springer: Boston, MA, USA, 1994.

5. Bazovsky, I. Reliability Theory and Practice; Prentice-Hall, Inc.: Englewood Cliffs, NJ, USA, 1961.

6. Billinton, R.; Allan, R.N. Reliability Evaluation of Engineering Systems: Concepts and Techniques, 2nd ed.; Plenum Press: New York, NY, USA, 1992.

7. Walski, M.; Chase, D.; Savic, D.; Grayman, W.; Beckwith, S.; Koelle, E. Advanced Water Distribution Modelling and Management; Haestad: Waterbury, CT, USA, 2003.

8. Mays, L.W.; Tung, Y.K. Hydrosystems Engineering and Management; McGraw-Hill: New York, NY, USA, 1992.

9. Walski, T.M. Practical aspects of providing reliability in water distribution systems. Reliab. Eng. Syst. Saf. 1993, 42, 13-19. [CrossRef] 
10. Kanakoudis, V.; Tolikas, D. Assessing the Performance Level of a water system. Water Air Soil Pollut. Focus 2004, 4, 307-318. [CrossRef]

11. Kanakoudis, V. Vulnerability based management of water resources systems. Hydroinformatics 2004, 6, 133-156.

12. Gargano, R.; Pianese, D. Reliability as Tool for Hydraulic Network Planning. J. Hydraul. Eng.-ASCE 2000, 126, 354-364. [CrossRef]

13. Khomsi, D.; Walters, G.A.; Thorley, A.R.D.; Ouazar, D. Reliability tester for water distribution networks. J. Comput. Civ. Eng. 1996, 10, 10-19. [CrossRef]

14. Giustolisi, O.; Savic, D.A.; Berardi, L.; Laucelli, D. An Excel-based solution to bring water distribution network analysis closer to users. In Proceedings of the Computer and Control in Water Industry (CCWI), Exeter, UK, 5-7 September 2011; Savic, D.A., Kapelan, Z., Butler, D., Eds.; Volume 3, pp. 805-810.

15. Berardi, L.; Laucelli, D.; Giustolisi, O. InnoWatING: Water Distribution Network Modelling of the Oppegård Municipality in Oslo; Research at Politecnico di Bari: General Report 2014, 1st Workshop on the State of Art and Challenges of Research Efforts at POLIBA 3-5 December 2014; University in Bari: Bari, Italy, 2014.

16. Giustolisi, O.; Kapelan, Z.; Savic, D.A. Extended period simulation analysis considering valve shutdowns. J. Water Resour. Plan. Manag. 2008, 134, 527-537. [CrossRef]

17. Giustolisi, O.; Savic, D.A. Identification of segments and optimal isolation valve system design in water distribution networks. Urban Water J. 2010, 7, 1-15. [CrossRef]

18. Berardi, L.; Ugarelli, R.; Røstum, J.; Giustolisi, O. Assessing mechanical vulnerability in water distribution networks under multiple failures. Water Resour. Res. 2014, 50, 2586-2599. [CrossRef]

19. Wengström, T.R. Comparative Analysis of Pipe Break Rates: A Literature Review. Publication 2:93; Department of Sanitary Engineering, Chalmers University of Technology: Gothenburg, Sweden, 1993.

20. Kanakoudis, V.; Tsitsifli, S.; Samaras, P.; Zouboulis, A.; Demetriou, G. Developing appropriate Performance Indicators for urban water supply systems evaluation across the Mediterranean. Water Util. J. 2011, 1, 31-40.

21. Kanakoudis, V.; Tsitsifli, S.; Samaras, P.; Zouboulis, A.; Banovec, P. A new set of water losses related Performance Indicators focused on areas facing water scarcity conditions. Desalin. Water Treat. 2013, 51, 2994-3010. [CrossRef]

22. Kanakoudis, V.; Tsitsifli, S.; Zouboulis, A. WATERLOSS project: Developing from theory to practice an integrated approach towards NRW reduction in urban water systems. Desalin. Water Treat. 2015, 54, 2147-2157. [CrossRef]

23. Kanakoudis, V.; Tsitsifli, S.; Cerk, M.; Banovec, P.; Samaras, P.; Zouboulis, A. Basic principles of a DSS tool developed to prioritize NRW reduction measures in water pipe networks. Water Qual. Expo. Health 2015, 7, 39-51. [CrossRef]

24. Bragalli, C.; Giansanti, F.; Zingali, L.; Montanari, A. Multi criteria decision analysis to set the priority of interventions in water distribution systems. In Proceedings of the 13th International Conference on Hydroinformatics-HIC2018, Palerm, Italy, 1-6 July 2018.

25. Gupta, R.; Bhave, P.R. Reliability analysis of water distribution systems. J. Environ. Eng. 1994, 120, 447-460. [CrossRef]

26. Tanymboh, T.T.; Tabesh, M.; Burrow, R. Appraisal of source head methods for calculating reliability of water distribution networks. J. Water Resour. Plan. Manag. 2001, 127, 206-213. [CrossRef]

27. Liserra, T.; Maglionico, M.; Ciriello, V.; Di Federico, V. Evaluation of reliability indicators for WDSs with demand-driven and pressure-driven models. Water Resour. Manag. 2014, 28, 1201-1217. [CrossRef]

28. Berardi, L.; Laucelli, D.B.; Simone, A.; Raspati, G.; Ugarelli, R.M.; Giustolisi, O. Mechanical reliability analysis of a real network to support the design of Isolation Valve System. In Proceedings of the Computing and Control for the Water Industry (CCWI2017), Sheffield, UK, 5-7 September 2017.

29. Wagner, J.M.; Shamir, U.; Marks, D.H. Water distribution reliability: Simulation methods. J. Water Res. Plan. Manag. 1988, 114, 276-294. [CrossRef]

30. ISO 31000:2009 Risk Management. Available online: https:/ /www.iso.org/obp/ui/\#iso:std:iso:31000:ed-1: v1:en (accessed on 28 September 2017). 
31. ISO Guide 73:2009 Risk Management-Vocabulary. Available online: https://www.iso.org/obp/ui/\#iso:std: iso:guide:73:ed-1:v1:en:term:3.7.1 (accessed on 28 September 2017).

32. Delibera Della Giunta Provinciale. Decreto del Presidente Della Provincia del 20/03/2006 n.12. Available online: http:/ / lexbrowser.provinz.bz.it (accessed on 8 June 2017). 